\title{
Low Probability of Intercept (LPI) Radar Signal Identification Techniques
}

\author{
M. Rajani Devi ${ }^{1}$, K. Ramanjaneyulu ${ }^{2}$ and B. T. Krishna ${ }^{3}$ \\ ${ }^{1}$ Vardhaman College of Engineering, Hyderabad, Telangana, India \\ ${ }^{2}$ PVP Siddhartha Institute of Technology, Vijayawada, Andhra Pradesh, India \\ ${ }^{3}$ JNTU Kakinada, Kakinada, Andhra Pradesh, India
}

\section{ABSTRACT}

A structure which a makes use of both Radar and Electronic Support tools is known as Low Probability of Intercept. Evading identification by ES receiver is the main aim behind the LPI radar. Features like frequency variability, low power, and wide bandwidth lend the property of Low probability of Intercept to the emitter making it undetectable by passive intercept devices like electronic intelligence receivers, radar warning, and electronic support. Novel signal processing methods are needed for identifying LPI radar waveforms. To retrieve meaningful information from the fed signal, Higher Order Spectral Analysis algorithms are utilized. Bispectrum, Bicoherence and Trispectrum techniques comprise the methodologies. For every LPI signal, the images generated by the algorithms are distinct and mean to be identifiers.

\section{KEY WORDS: LOW PROBABILITY OF INTERCEPT (LPI), RADAR WARNING, FREQUENCY VARIABILITY.}

\section{INTRODUCTION}

Low Probability of Intercept is recommended as a prudent strategy by several radar customers nowadays. Features like frequency variability, low power, and wide bandwidth lend the property of Low probability of Intercept to the emitter making it undetectable by passive intercept devices like electronic intelligence receivers, radar warning, and electronic support. Long-range detection is the goal behind LPI radars instead of shorter ranges. The viability of an LPI radar is directly proportional to the difficulty of receiver identifying the radar emitter's traits.

In the wake of progressively advanced intercept receivers which identify radar emitters, an LPI criteria is being preferred. The interception of radar transmission could instantly result in electronic attack in implementations like surveillance, altimeters, and navigation. In addition, the possible chance of attack by ARMs and military weapons makes it necessary for an LPI emitter. This

Biosc Biotech Res Comm P-ISSN: 0974-6455 E-ISSN: 2321-4007

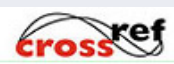

Identifiers and Pagination

Year: 2021 Vol: 14 No (5) Special Issue

Pages: $365-373$

This is an open access article under Creative

Commons License Attribn 4.0 Intl (CC-BY).

DOI: http://dx.doi.org/10.21786/bbrc/14.5/63 property of pre-empting interception safeguards these emitters against almost all threats. Intercepting receivers face problems when making use of just power spectral analysis while attempting to detect and/or intercept LPI radars which generally utilize wideband $\mathrm{CW}$ signals thereby making it impossible to intercept and/or detect. This leaves them with no choice but to utilize advanced methods and signal processing systems required to make the appropriate electronic attack.

The differentiation of four intercept receiver signal processing methods to identify LPI wave metrics is performed in this work. Several kinds of LPI CW waves were produced with $\mathrm{S} / \mathrm{N}$ ratios of 0 and $-6 \mathrm{~dB}$ for evaluating the four methods. FMCW, P1 through P4, Frank code, Costas hopping and combined PSK/FSK are LPI waveforms which were produced. Filter bank processing with higher order statistics, Wigner distribution, Quadrature mirror filter banks, and Cyclostationary processing are the signal processing methods upon which the comparison was performed.

In implementations like underwater acoustics, communications, and seismic surveillance, the requirement to identify transient signals crops up. The optimal detector comprises a matching filter and a threshold circuit whose threshold is set to best perform a function when the arrival time and waveform of the signal is given. Many subpar methods for identification exist. For only a known set of signals and high SNR assumptions, techniques having

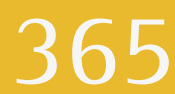


basis in short-time Gabor transform, Fourier transform, wavelet transform and non-linear methods work fine.

Although it is not feasible in practice, the matched filter technique makes it necessary that the waveshape and arrival time are provided. A match filter cannot be developed in applications without the knowledge of arrival time and waveshape of transient signal. A transient sound like a knock sound or a hydro sound wave is a case in point, which don't have a clear waveform. A transient sound like the sound of a knock or hydroacoustic pulse lacking a pronounced waveshape would be an instance of such a case. Moreover, from the same origin, the waveshape can change depending on the event. It is therefore difficult for these transient signals to develop a matched filter.

2. Signal Processing Techniques: Following are the signal processing methods for identifying LPI radar properties.

2.1 Filter Bank and Higher Order Statistics: As demonstrated in the figure below, the Filter Bank and Higher Order Statistics method has basis in the utilization of a parallel array of filters and higher order statistics. The main objective behind the filter bank is to segment the input signal in small frequency bands, giving an entire time frequency overview. Following this, every band is performed on separately and is then fed to a third-order estimator which reduces noise. After identifying LPI radar properties, they can be utilized to make an attack on the LPI radar.

Identifying a transient signal s(n) in an individual noisy signal $x(n)$ is the primary goal. For the received signal, the signal including the noise is taken into account i.e.

$$
x(n)=s(n)+v(n)
$$

Figure 1: The filter bank and higher order statistic technique

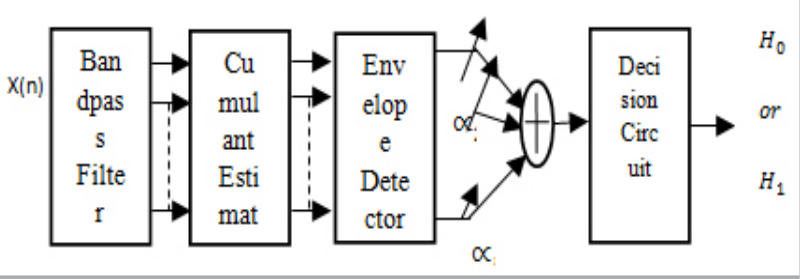

Signal s(n) is a transient sign of unfamiliar waveform and time of arrival; $\mathrm{v}(\mathrm{n})$ is a non-familiar symmetrical probability distribution color noise additive. Let us take into account the hypothesis test given below.

$$
\begin{aligned}
& H 0: x(n)=v(n) \\
& H 1: x(n)=s(n)+v(n)
\end{aligned}
$$

The hypothesis H1proves the existence of signal and noise compared to the hypothesis Ho, which only has noise.
Wigner Distribution: The Wigner distribution [9] is a relatively productive method for the analysis of timefrequency for LPI waveform property. The Wigner W $\mathrm{x}$ $(\mathrm{t}, \mathrm{w})$ distribution is shown.

$$
W_{x}(t, w)=x\left(t+\frac{T}{2}\right) x^{*}\left(t-\frac{T}{2}\right) e^{-j w T} d T
$$

In this case $\mathrm{t}$ is a time variable, $\mathrm{w}$ is a frequency variable, and ${ }^{*}$ is a complex combination. A two-dimensional function which is in terms of frequency content of signal with respect to time, and has several attributes like:

1) Real nature.

2) It contains marginal distributions:

$$
\begin{aligned}
& \int_{-\infty}^{+\infty} W_{x}(t, w)=|X(w)|^{2} \\
& \frac{1}{2 \pi} \int_{-\infty}^{+\infty} W_{c}(t, w) d w=|x(t)|^{2}
\end{aligned}
$$

Here X ( U ) be the Fourier transform of ( $t$ ) ;

3) Two important functions - the instantaneous frequency and group delay - are provided by their first time in relation to $t$ and $w$.

4) Lack of intermingling of time and frequency resolutions. It is demonstrated that the windowed Wigner distribution [10] can be expressed as

$$
W(t, w)=\frac{1}{2 \pi} \int_{-\infty}^{+\infty} W_{x}(t, \epsilon) W_{w}(0, w-\epsilon) d \epsilon
$$

Figure 2: two-channel QMF bank-based sub band codec

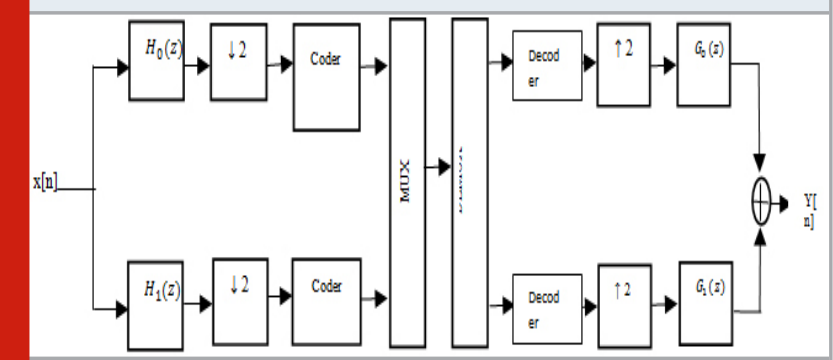

Figure 3: cyclostationary modeling

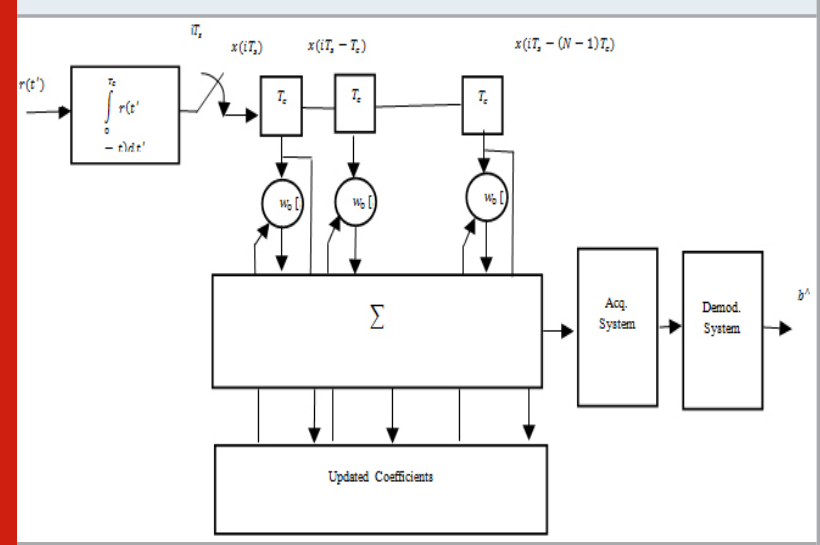

Here $\mathrm{W}(\mathrm{t}, \mathrm{w})$ is the pseudo-Wigner distribution, and $\mathrm{W}_{\mathrm{x}}$ $(t, w)$ and $W_{w}(t, w)$ are the Wigner distributions of the 
signal $\mathrm{x}(\mathrm{t})$ and the window $\mathrm{w}(\mathrm{t})$ correspondingly. The convolution of two Wigner distributions in the frequency domain is $\mathrm{W}(\mathrm{t}, \mathrm{U})$ shown by the final equation. Hence, the time resolution is constant, while the frequency resolution is impacted by windowing. Requiring only some amount of processing to derive the kernel function, the Wigner Distribution can effectively obtain the waveform parameters.

Quadrature mirror filter bank: A discrete-time signal $x[n]$ is segmented into a number of sub-band signals with the help of an analysis filter bank in several applications. After this, the sub-band signals are processed. Consequently, an output signal $y[n]$ is obtained by merging the processed sub-band signals with the help of a synthesis filter bank. They can be downsampled before processing if the sub band signals are band confined to frequency ranges significantly lesser than those of the actual input signal $\mathrm{x}[\mathrm{n}]$. The sub-band signals could be down-sampled before processing if they are confined to frequency rangers lesser than the initial input signal $x[n]$. The handling of the downsampled signals is performed more productively due to the low sampling rate. . Following this, before being merged together as a signal by the synthesis filter bank, the signals are up-sampled. These signals are later upsampled and then merged with the help of synthesis filter bank together as a signal. The merged arrangement is known as quadrature-mirror filter (QMF) bank.

By selecting the relevant filters, the output $y[n]$ could possess some or all properties of the input signal $\mathrm{x}[\mathrm{n}]$ if the down-sampling and up-sampling factors are the same or exceed the number of bands of the filter bank. The arrangement is called a critically sampled filter bank if the up-sampling and down-sampling factors are same as the number of bands. This arrangement is widely used in coding of a signal $x[n]$. Following illustration shows the basic two-channel QMF bank-based sub band codec (coder/decoder).

Cyclo-stationary spectral analysis: Digitally modulated signals are cyclostationary. By definition, the probabilistic parameters like correlation or mean value, change with respect to time with single or multiple periodicity. In addition, this property is also the fact that the signal is linked to frequency shifts in frequency shifts. Benefit in the examination of LPI waveforms making use of cyclostationary modeling, is that non-zero correlation is observed between certain frequency components when their frequency gap is in reference to the periodicity of interest. The magnitude of that frequency gap is called the cycle frequency. In an illustration of the Spectral Correlation Density (SCD) function the spectral correlation properties of a signal are clear.
3.1 Graphical User Interface (GUI): The 10U (5000 samples) LPI signals produced with software for examining purposes (at IF level of $160 \mathrm{MHz}$, Sampled at $500 \mathrm{MHz}$ ), a GUI has been designed. The following signals are considered

- Barker

- Polyphase codes

- Frank

- Signal with no modulation

3.1.1 Bispectrum: Within the field of data analysis, the bispectrum is a piece of data for locating nonlinear interactions. conventional power spectrum. The Fourier transform of $\mathrm{C} 3(\mathrm{t} 1, \mathrm{t} 2)$ is the bispectrum or bispectral density (third-order cumulant-generating function). Implementing the convolution theorem permits rapid computation of bispectrum.

$$
B\left(f_{1}, f_{2}\right)=X^{*}\left(f_{1}+f_{2}\right) \cdot X\left(f_{1}\right) \cdot X\left(f_{2}\right)
$$

They belong to the group of higher-order spectra or polyspectra and provide the power spectrum with additional information. The polyspectrum of the 3rd order is more commonly known in calculations. Statistics of the second order relate to the mean $m$ value and variance. They are expressed as the operation of the expected value and $\mathrm{X}$ is the result of the altered method:

$$
\begin{gathered}
m_{X}=E\{X\} \\
\sigma_{X}^{2}=E\left\{\left(X-m_{X}\right)^{2}\right\}
\end{gathered}
$$

If $\mathrm{x}$ is a discrete time signal, then the second order moment, or ACF, is shown as.

$$
m_{X}^{2}(i)=E\{X(n) \cdot X(n+i)\} .
$$

Besides these, statistics from a higher order provide more orderly moments $(\mathrm{m} 3, \mathrm{~m} 4, \ldots)$ and nonlinear, so-called cumulative, higher order combinations (c1, c2, c3, ...).

3.1.2 Bicoherence: A squared, normalized form of the bispectrum is Bicoherence. The bicoherence obtains values in the range of 0 and 1 , thereby an easy metric for measuring the amount of phase coupling in a signal. Also referred to as bispectral coherency. The prefix bi- in bispectrum and bicoherence means two frequencies of an individual signal and not two time series $\mathrm{xt}$, yt. It is different when calculating coherence, since the input and output values are necessary when two auto spectra and one cross-spectrum are approximated. Bicoherence, meaning it can be derived from an individual signal, is an auto quantity. The coherence function offers a measurement of variations by linearity in the system between the input and output devices. The bicoherence calculates the signal energy in quantity at only the quadratically combined bifrequencies. Non-linear process 
are not covered properly by second order statistics. The quadratic phase coupling problem is a known issue. The supposition that a signal $\mathrm{x} / \mathrm{n}$ is filtered through a system with quadratic characteristics has two harmonics at frequencies $\mathrm{f} 1$ and $\mathrm{f} 2$;

e.g. $y(n)=[x(n)] 2, y(n)$ has harmonics at the frequencies $\mathrm{f} 1, \mathrm{f} 2, \mathrm{f} 1+\mathrm{f} 2$ and $\mathrm{f} 1-\mathrm{f} 2$.

3.1.3 Tri spectrum: The trispectrum is a data piece for finding non-linear interactions. The traditional power spectrum is the Fourier transformation of the cumulative secondary order, which is also known as autocorrelation function. The Fourier transform of $\mathrm{C} 4(\mathrm{t} 1, \mathrm{t} 2, \mathrm{t} 3)$ is the trispectrum or trispectral density (fourth-order cumulantgenerating function). The trispectrum $\mathrm{T}(\mathrm{f} 1, \mathrm{f} 2, \mathrm{f} 3)$ belongs to the higher order spectrum group and provides further power spectrum data. It's a 3D system. The scope of the maximum kurtosis phase estimate used for deconvolution of seismic data to determine the layer structure has been determined.

Figure 4: Barker 5 signal (4 pulses)

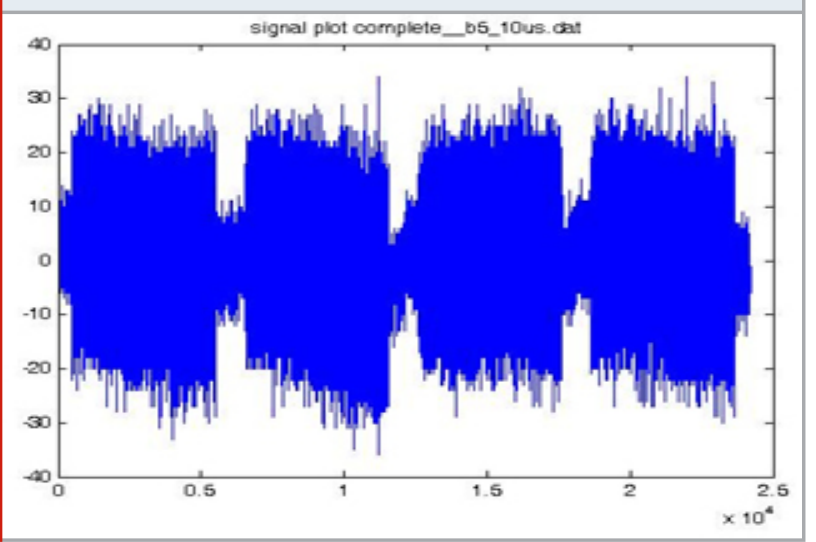

Figure 5: Barker 5 - Bispectrum

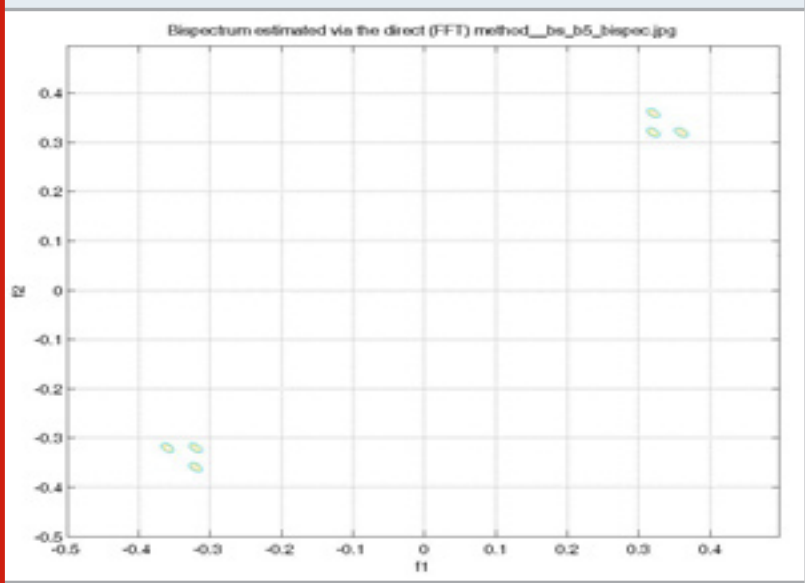

RESULTS

A GUI has been developed to analyze the LPI signals (at IF level of $160 \mathrm{MHz}$, Sampled at $500 \mathrm{MHz}$ ) of 10uS duration
(5000 samples) generated from the Signal Source / samples generated through software. The following signals are considered Barker, Polyphase codes, Frank and Signal with no modulation. These signals are analyzed with three algorithms such as Bispectrum, Bicoherence and Trispectrum in the presence of noise.

Figure 6: Barker - 5 Bicoherence

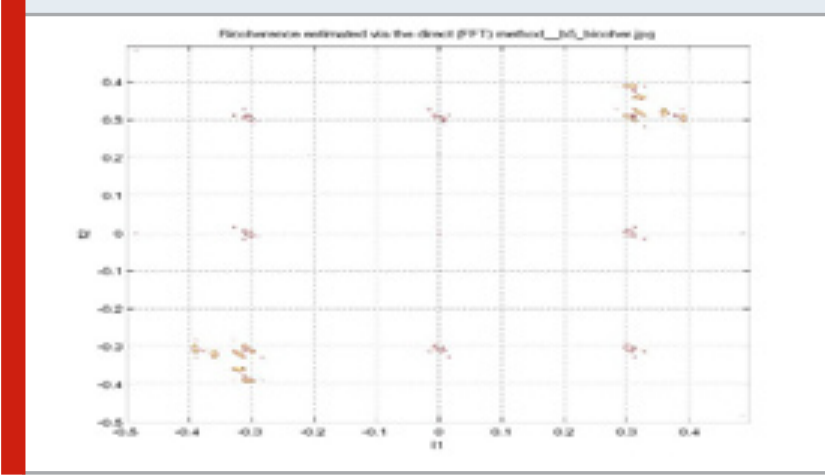

Figure 7: Barker-5 Trispectrum

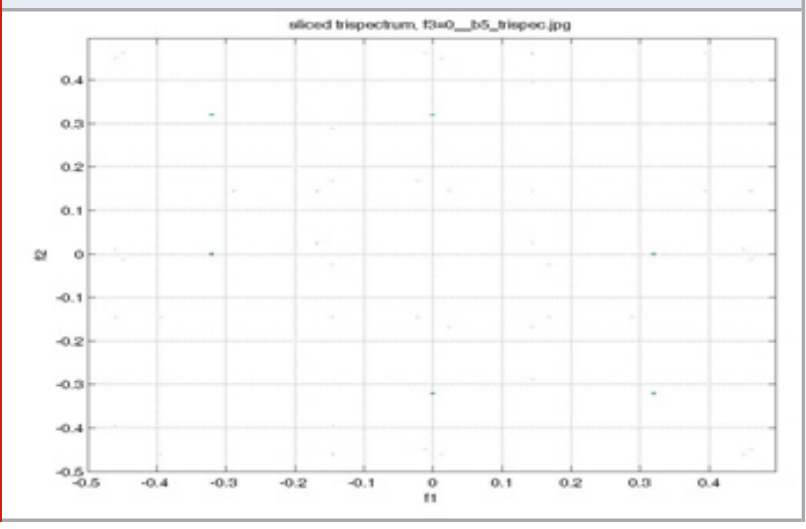

Figure 8: Barker 5 (3dB Noise)

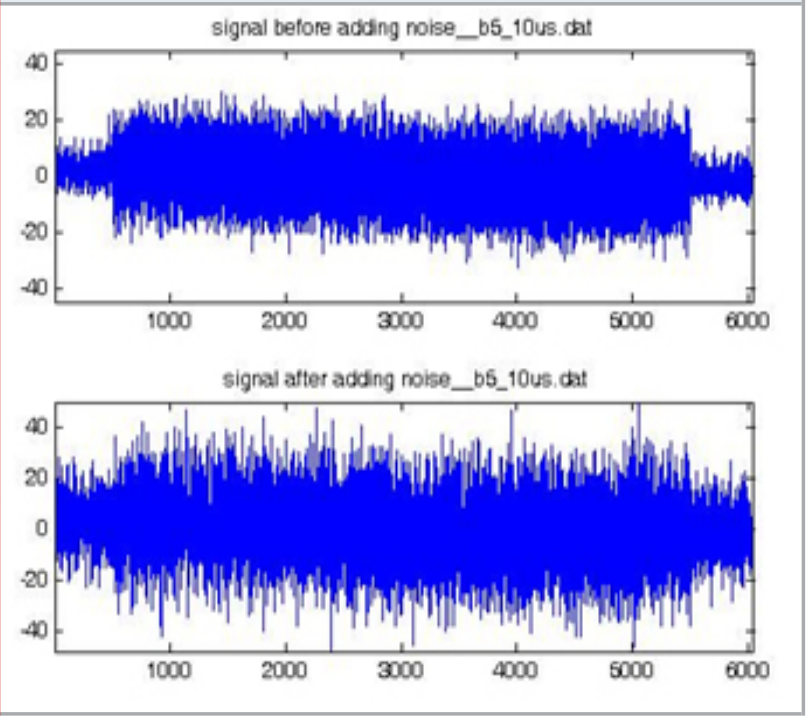


Figure 9: Barker-5 Bispectrum with 3dB Noise

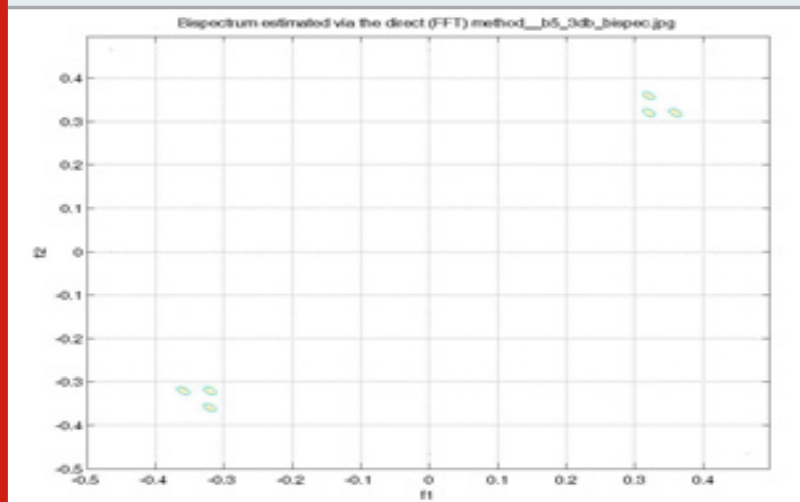

Figure 10: Barker-5 Bicohorence with 3dB Noise

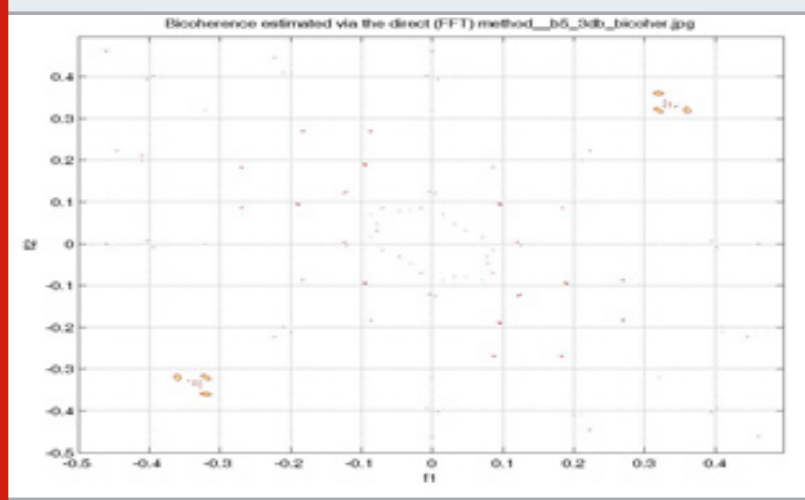

Figure 11: Barker-5 Trispectrum with $3 \mathrm{~dB}$ Noise

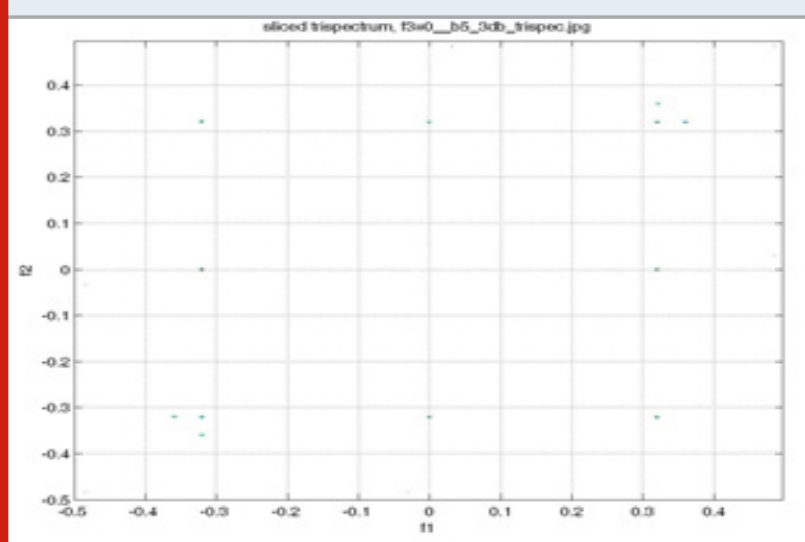

Figure 12: Barker 5 -3dB Noise

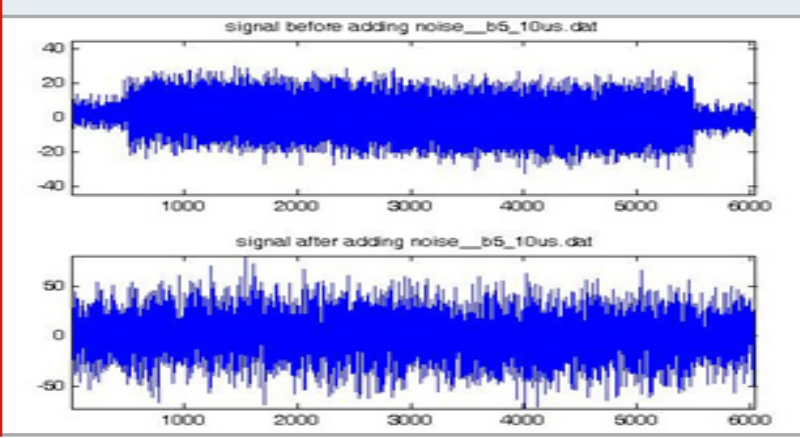

Figure 13: Barker-5 Bispectrum with $-3 \mathrm{~dB}$ Noise

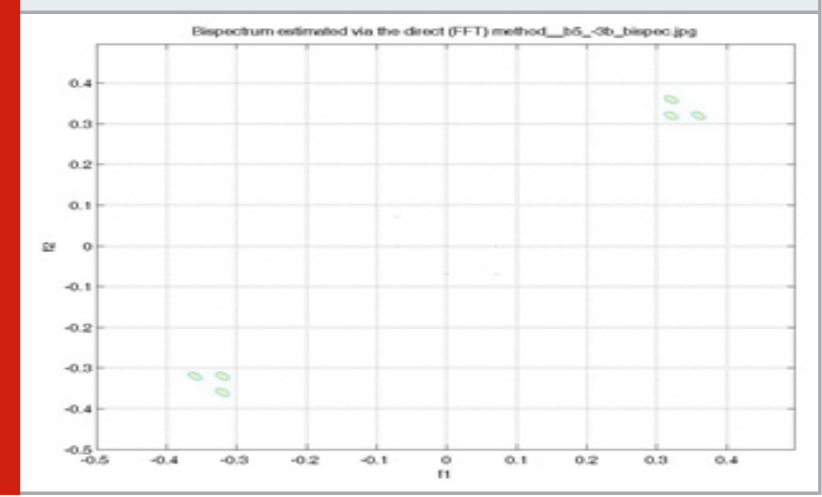

Figure 14: Barker-5 Bicohorence with -3 dB Noise

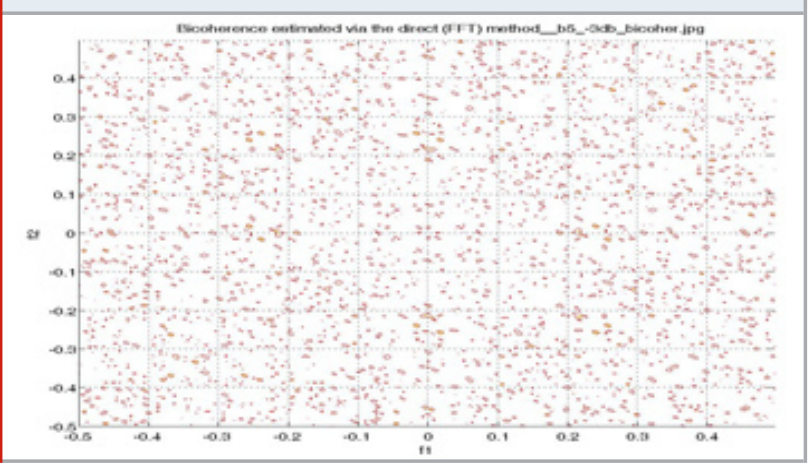

Figure 15: Barker-5 Trispectrum with $-3 \mathrm{~dB}$ Noise

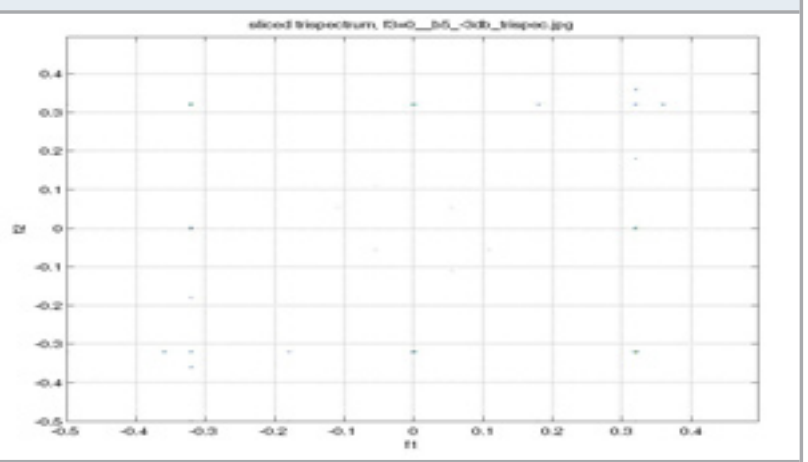

figures 9 to 15 shows the analysis of bakers 5 signal with three different algorithms in the presence guassian noise with 3Dband -3db.from the above figures we observe that the Trispectrum computation is more complex than others.

P1 Signal( Poly phase code):

Figures 21 to 25 shows the analsis of polyphase codes with three different algorithms in the presence of Gaussian noise $3 \mathrm{db}$ and $-3 \mathrm{db}$.from the figures it is observed that non-linear interactions can be observed clearly in Trispectrum in the presence of noise. 
Figure 16: P1 Signal (4 pulses)

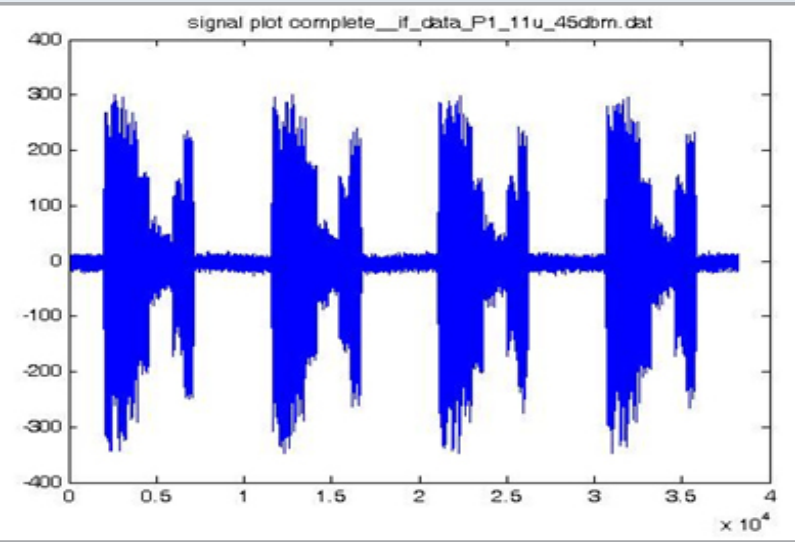

Figure 17: P1 Bispectrum

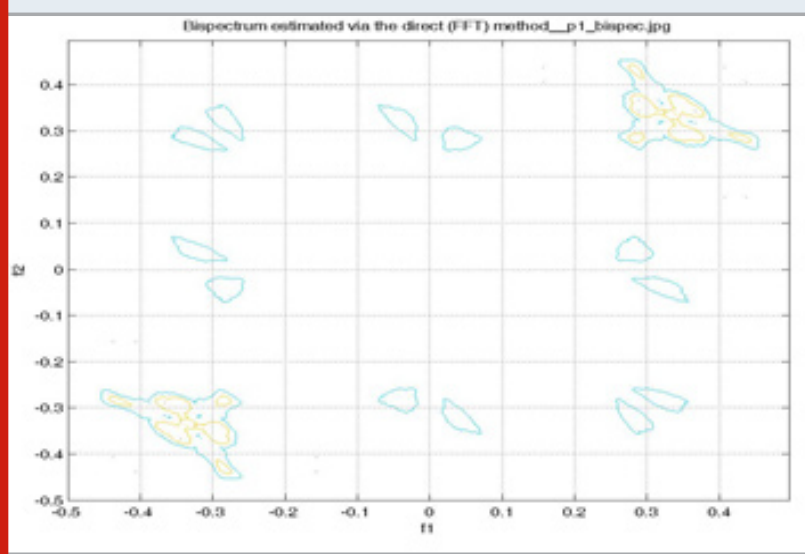

Figure 18: P1 Bicohorence

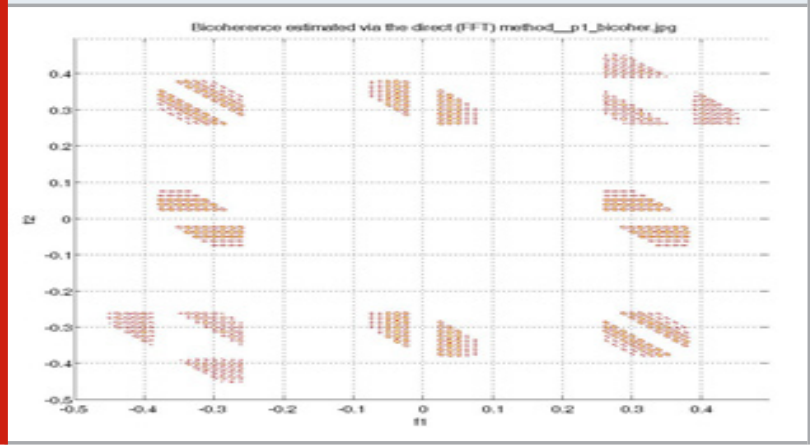

Figure 19: P1 Trispectrum

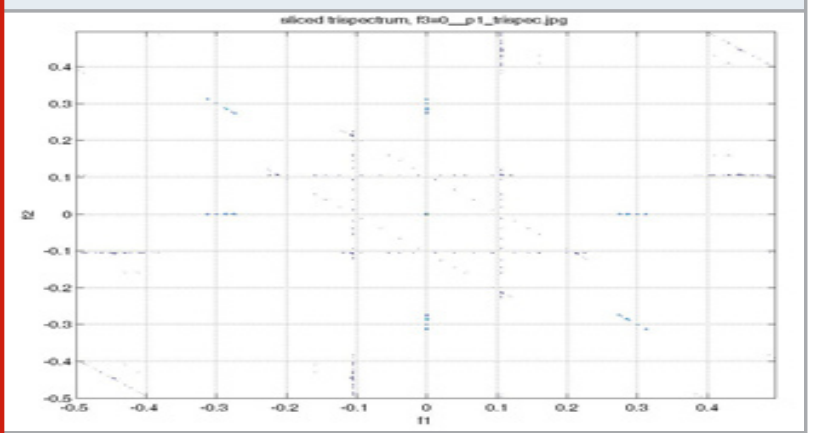

Figure 20: P1 with 3 dB Noise

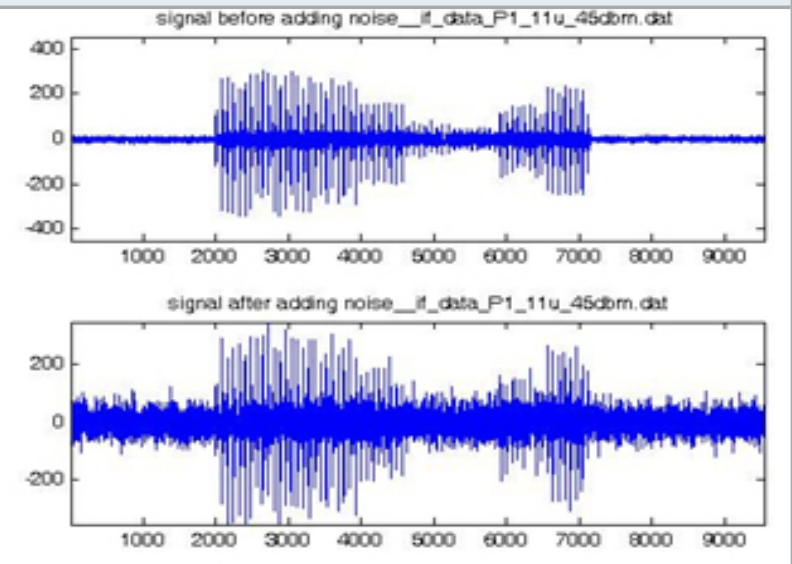

Figure 21: P1 Bispectrum with $3 \mathrm{~dB}$ Noise

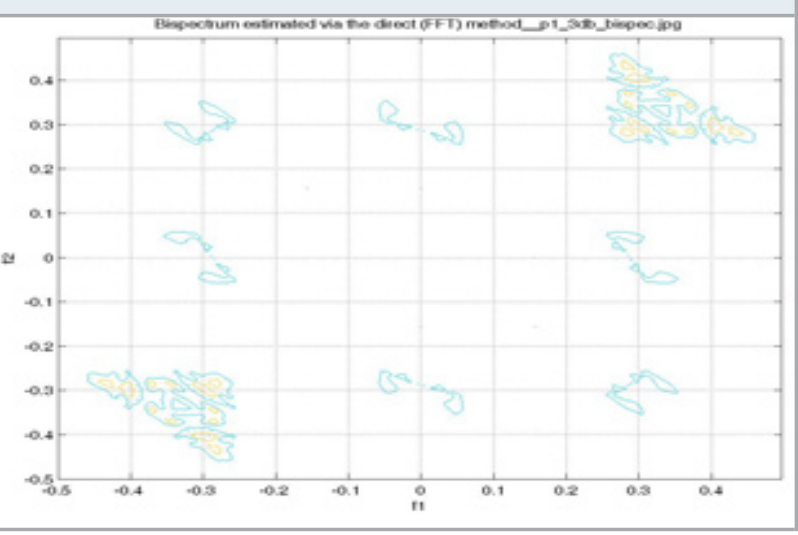

Figure 22: P1 Bicohorence with $3 \mathrm{~dB}$ Noise

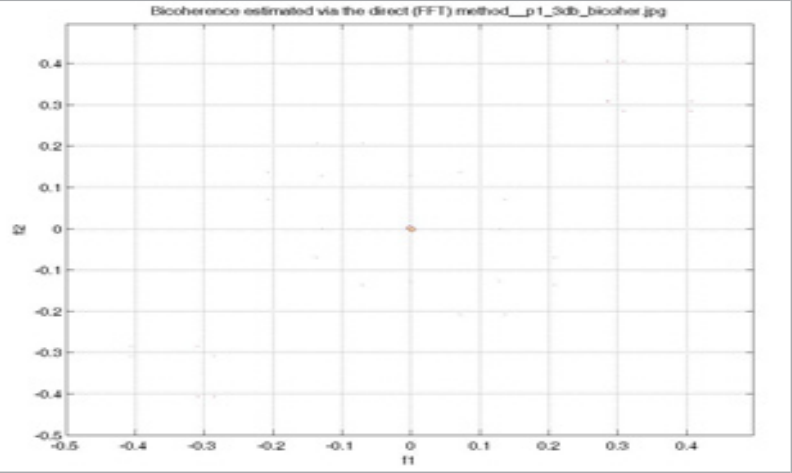

Figure 23: P1 Trispectrum with $3 \mathrm{~dB}$ Noise

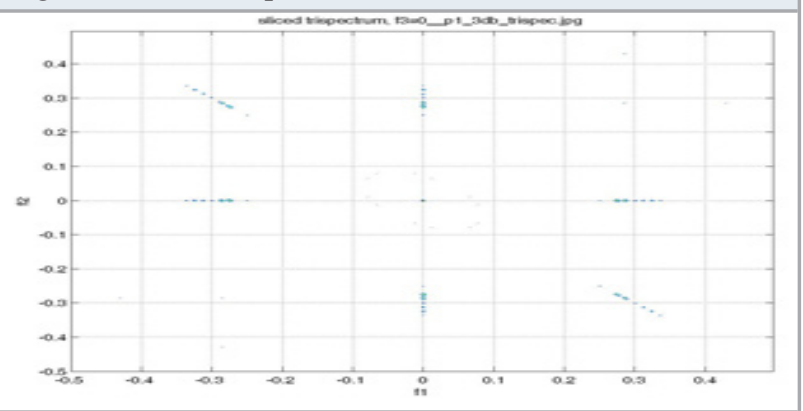




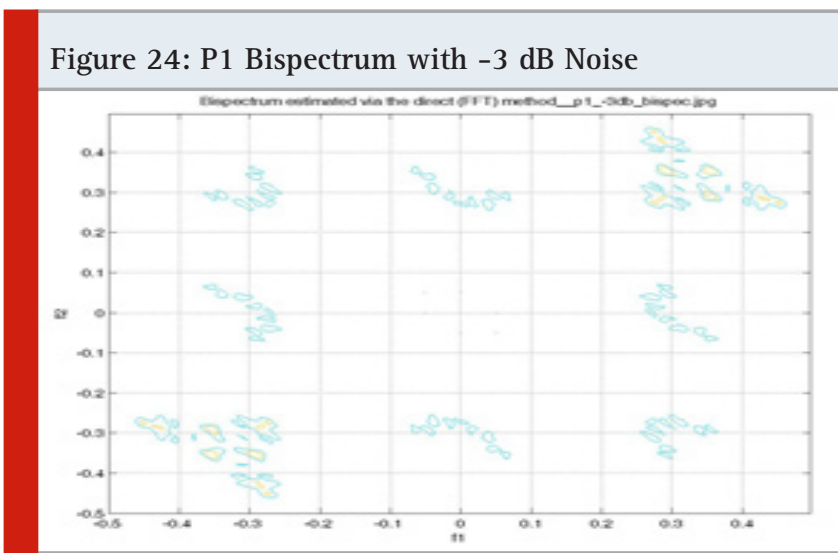

Figure 25: P1 Trispectrum with $-3 \mathrm{~dB}$ Noise

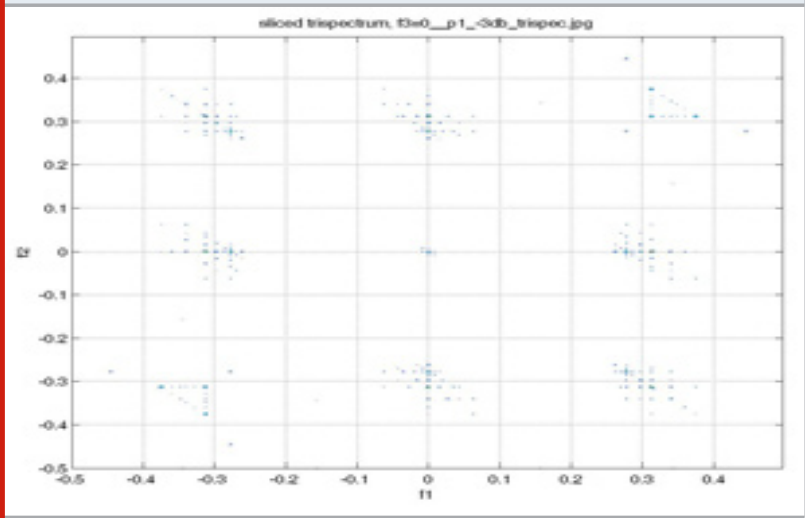

Figures 26 to29 shows the analsis of no modulation signal with three different algorithms in the presence of Gaussian noise $3 \mathrm{db}$ and $-3 \mathrm{db}$.from the figures it is observed that the three methods can differentiate the signals. Frank Signal:

Figure 26: No-mod Signal (4 pulses)

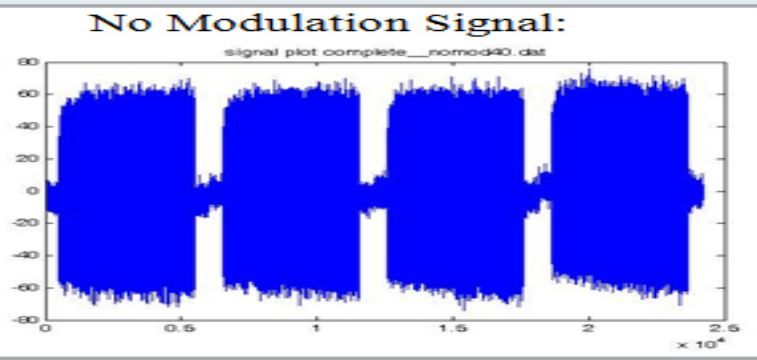

Figure 27: No-mod Signal Bispectrum

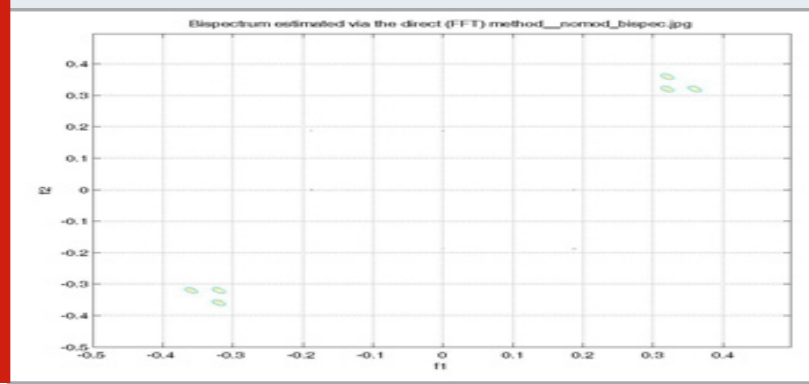

Figure 28: No-mod Signal Bicohorence

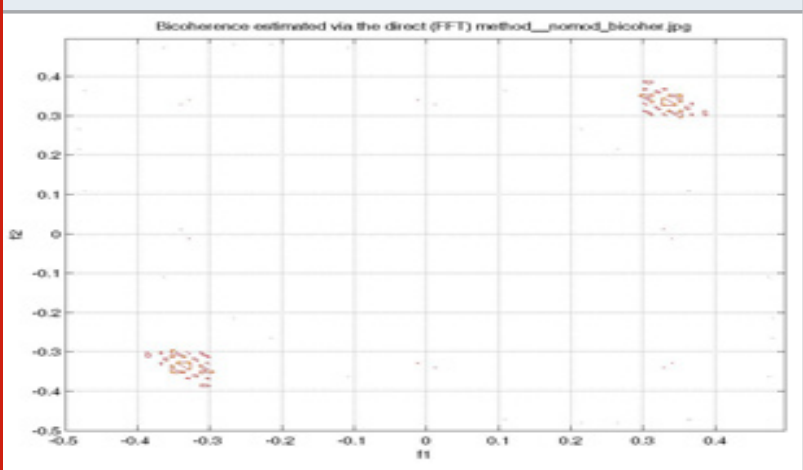

Figure 29: No-mod Signal Trispectrum

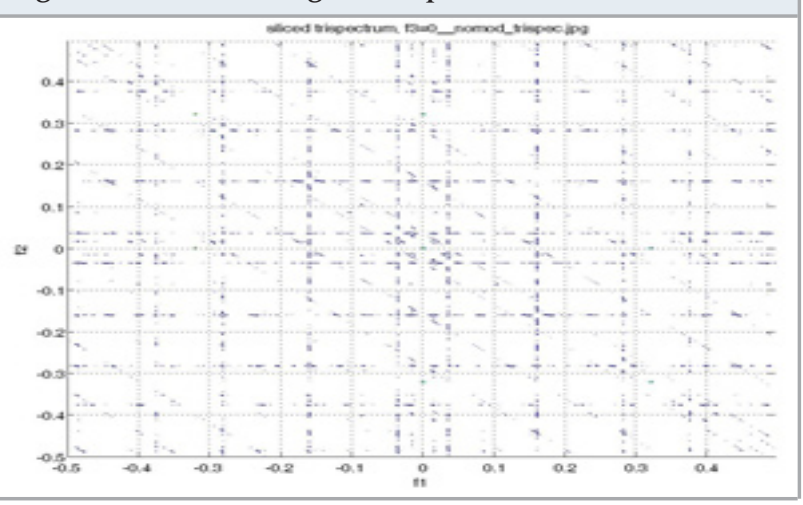

Figure 30: Frank Signal (4 pulses)

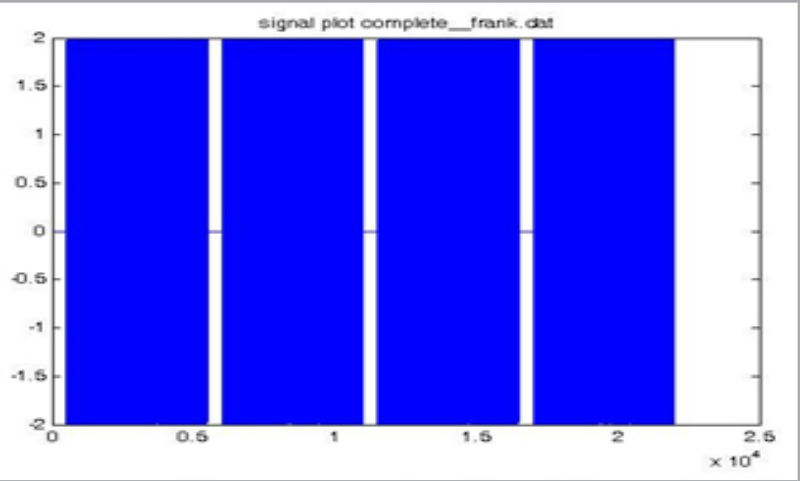

Figure 31: Frank Signal Bispectrum

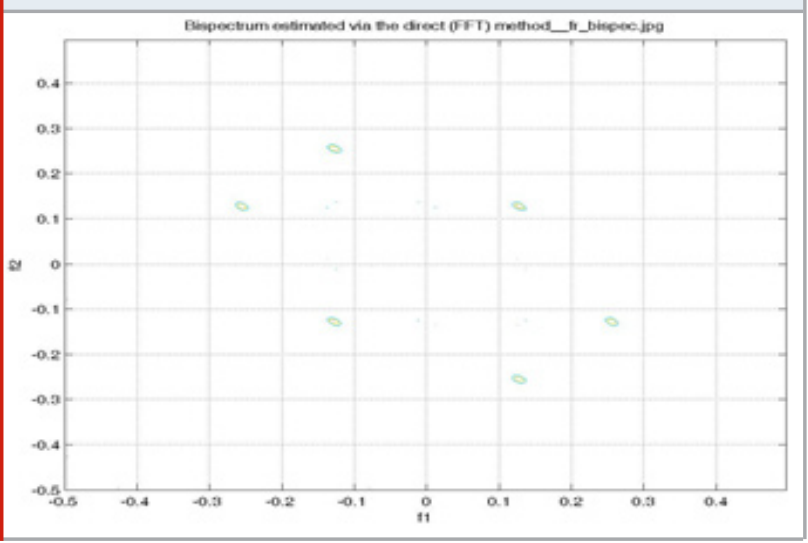


Figure 32: Frank Signal Bicohorence

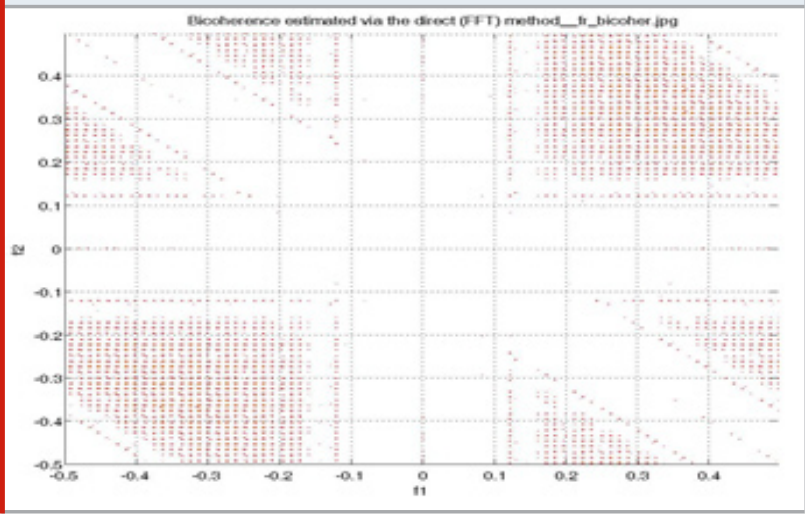

Figure 33: Frank Signal Trispectrum

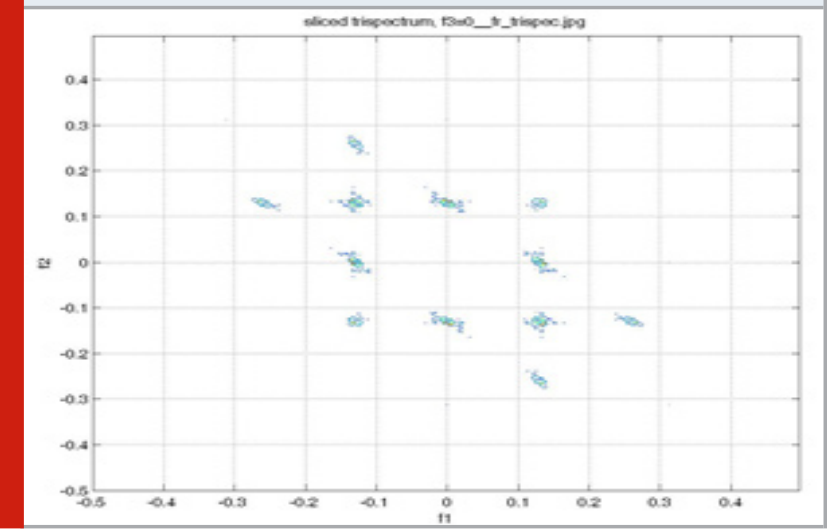

Figures 30 to 33 shows the analsis of frank signal with three different algorithms in the presence of Gaussian noise $3 \mathrm{db}$ and $-3 \mathrm{db}$.from the figures it is observed The graphs produced and given above are unique and are of help in detecting and distinguishing signals at certain noise levels.

\section{CONCLUSION}

With the help of higher order spectral analysis, the signals can be distinguished from one another. Trispectrum calculation is relatively difficult. In spite of calculated Gaussian noises of order upto $-6 \mathrm{db}$, Bispectrum, bicoherence and trispectrum can differentiate the signals. All three techniques are applied while classifying signals impacted by Gaussian noise. That can be very helpful in identifying and distinguishing LPI radar signals. The graphs produced and given above are unique and are of help in detecting and distinguishing signals at certain noise levels. Some existing and potential academic topics in the field of the higher-order spectrum are non-stationary and cyclostationary signal analysis with HOS-based time-frequency representatives, HOS-based wavelet representations and higher-order statistics used to examine chaotic signals.

\section{REFERENCES}

Abdullah, Abdul Rahim Bin, Ahmad Zuri Bin Sha'ameri, and Auzani Bin Jidin. "Classification of power quality signals using smooth-windowed Wigner-Ville distribution." In 2010 International Conference on Electrical Machines and Systems, pp. 1981-1985. IEEE, 2010.

Agrawal, S. K., and O. P. Sahu. "Two-channel quadrature mirror filter bank: An overview.” International Scholarly Research Notices 2013 (2013).

Båth, B. M. Spectral analysis in geophysics. Elsevier, 2012.

Heath, Robert W., Nuria Gonzalez-Prelcic, Sundeep Rangan, Wonil Roh, and Akbar M. Sayeed. "An overview of signal processing techniques for millimeter wave MIMO systems." IEEE journal of selected topics in signal processing 10, no. 3 (2016): 436-453.

Kishore, Thokala Ravi, and K. Deergha Rao. "Automatic intrapulse modulation classification of advanced LPI radar waveforms." IEEE Transactions on Aerospace and Electronic Systems 53, no. 2 (2017): 901-914.

Kocamis, Mehmet Burak, Hakan Abacı, Safak Bilgi Akdemir, Sertan Varma, and Alper Yildirim. "Deinterleaving for radar warning receivers with missed pulse consideration." In 2016 European Radar Conference (EuRAD), pp. 225-228. IEEE, 2016.

Punchihewa, Anjana, Qiyun Zhang, Octavia A. Dobre, C. Spooner, Sreeraman Rajan, and R. Inkol. "On the cyclostationarity of OFDM and single carrier linearly digitally modulated signals in time dispersive channels: Theoretical developments and application." IEEE Transactions on Wireless Communications 9, no. 8 (2010): 2588-2599.

Shevgunov, Timofey, Evgeniy Efımov, and Dmitriy Zhukov. "Averaged absolute spectral correlation density estimator." In 2018 Moscow Workshop on Electronic and Networking Technologies (MWENT), pp. 1-4. IEEE, 2018.

Shi, Chenguang, Fei Wang, Mathini Sellathurai, and Jianjiang Zhou. "Low probability of intercept based multicarrier radar jamming power allocation for joint radar and wireless communications systems." IET Radar, Sonar \&t Navigation 11, no. 5 (2016): 802-811.

Shi, Chenguang, Fei Wang, Mathini Sellathurai, Jianjiang Zhou, and Sana Salous. "Low probability of intercept-based optimal power allocation scheme for an integrated multistatic radar and communication system.” IEEE Systems Journal 14, no. 1 (2019): 983994.

Stamenkovic, Negovan, Dragana Živaljcvic, Vidosav Stojanovic, and Ivan Krstic. "The implementation of two channel IIR quadrature mirror filter bank based 
on residue arithmetic.” In 2014 18th International Symposium on Electrical Apparatus and Technologies (SIELA), pp. 1-4. IEEE, 2014.

Stankovic, Ljubisa, Srdjan Stankovic, and Milos Dakovic. "From the STFT to the Wigner distribution [lecture notes].” IEEE Signal Processing Magazine 31, no. 3 (2014): 163-174.

Wang, Ruiping, Huimin Guo, Larry S. Davis, and Qionghai Dai. "Covariance discriminative learning:
A natural and efficient approach to image set classification.” In 2012 IEEE conference on computer vision and pattern recognition, pp. 2496-2503. IEEE, 2012.

Zou, Jianbin, Kai Gao, Shujun Lu, and Eryang Zhang. "Coherent decoys jamming anti-radiation missiles." In 2013 IEEE Jordan Conference on Applied Electrical Engineering and Computing Technologies (AEECT), pp. 1-5. IEEE, 2013. 